\title{
Micro-accelerometer Based on Vertically Movable Gate Field Effect Transistor
}

\author{
Heung Seok Kang $\cdot$ Kang-Hee Lee $\cdot$ Dong-Youk Yang $\cdot$ Byoung Hee You $\cdot$ In-Hyouk Song
}

Received: 26 January 2015 / Accepted: 23 March 2015/Published online: 6 May 2015

(c) The Author(s) 2015. This article is published with open access at Springerlink.com

\begin{abstract}
A vertically movable gate field effect transistor (VMGFET) is proposed and demonstrated for a microaccelerometer application. The VMGFET using air gap as an insulator layer allows the gate to move on the substrate vertically by external forces. Finite element analysis is used to simulate mechanical behaviors of the designed structure. For the simulation, the ground acceleration spectrum of the 1952 Kern County Earthquake is employed to investigate the structural integrity of the sensor in vibration. Based on the simulation, a prototype VMGFET accelerometer is fabricated from silicon on insulator wafer. According to current-voltage characteristics of the prototype VMGFET, the threshold voltage is measured to be $2.32 \mathrm{~V}$, which determines the effective charge density and the mutual transconductance of $1.545 \times 10^{-8} \mathrm{C} \mathrm{cm}^{-2}$ and $6.59 \mathrm{~mA} \mathrm{~V}{ }^{-1}$, respectively. The device sensitivity is $9.36-9.42 \mathrm{mV} \mathrm{g}^{-1}$ in the low frequency, and the first natural frequency is found to be $1230 \mathrm{~Hz}$. The profile smoothness of the sensed signal is in $3 \mathrm{~dB}$ range up to $1 \mathrm{kHz}$.
\end{abstract}

Keywords MEMS · Micro-accelerometer - Suspended gate FET · Vertically movable gate FET · VMGFET

\section{Introduction}

The advancement of technology to make micro-mechanical structure using semiconductor fabrication processes leads the application of electrical components including transistors, capacitors, and inductors for microelectromechanical systems (MEMS) [1-6]. In terms of integration with electronics, silicon-based MEMS have gained much attention due to the potential applications in wireless communication system, mobile device, structural health monitoring system,

H. S. Kang - K.-H. Lee

Korea Atomic Energy Research Institute, 150 Deokjin-dong,

Yuseong-gu, Daejeon, Republic of Korea

D.-Y. Yang

LOGTECH Co. Ltd., 914 Gumgang Hightech Valley 1st,

Sungnam-si, Gyeonggi-do, Republic of Korea

B. H. You · I.-H. Song $(\bowtie)$

Department of Engineering Technology, Texas State University,

601 University Dr., San Marcos, TX 78666, USA

e-mail: in-hyouk.song@txstate.edu etc. [6-8]. One of the active electronic devices for sensing elements is movable gate field effect transistors (FET), whose gate is released from the substrate and free to move [9-12]. The MOSFET-type device contains dielectric layer to electrically insulate between gate electrode and substrate embedding source and drain of FET. The movable gate FET uses air gap as an insulator or a dielectric layer of the FET. The use of air layer allows the gate to move laterally along the channel of FET or vertically to the substrate by external forces such as acceleration or pressure. The former is called the laterally movable gate FET (LMGFET), and the latter is called the vertically movable gate FET (VMGFET) [12, 13]. The oscillation frequency of the sensing element is simply converted to electrical output without additional circuitry. In this paper, a micro-accelerometer using VMGFET is introduced, applicable for low-frequency vibration measurement $(<500 \mathrm{~Hz})$.

The state-of-the art technologies for low-frequency vibration sensing applications have been mostly used for capacitive or piezoresistive sensing scheme [14-16] while the research of VMGFET has been more focused on 
pressure/strain sensors or gas sensors $[2,10]$. In order to enhance the sensitivity to vertical motion using VMGFET, Aoyagi proposed nickel proof mass on flexible beam, which required alignment and electroplating to form proof mass [17]. The VMGFET in this paper is demonstrated and characterized using silicon on insulator (SOI) wafer for low-frequency vibration measurement. The resonant structure including moving gate of VMGFET is designed with meander-type beams to improve flexibility in vertical direction, and the long-channel device is used to increase the device sensitivity.

VMGFET is fabricated using standard IC fabrication processes such as UV lithography and silicon dry etching process. Silicon-based suspended microstructures with high quality factors can be applied for frequency-sensitive systems such as accelerometer and gyroscope integrated with electronic systems [18, 19]. The silicon-based process benefits mass production through batch fabrication, and compactness due to small size and IC compatibility.

The structural device layer serves as suspension gate and proof mass to induce the movement due to acceleration. Figure 1 is the schematic of the VMGFET accelerometer with a cross-sectional view, which illustrates a serpentine gate structure design with fixed anchors at the ends. The VMGFET structure is obtained by forming an air gap and using $\mathrm{SiO}_{2}$ as an insulator layer. The air gap allows the suspended gate to move vertically. The displacement of the gate modulates the charge distribution in the semiconductor and the change in capacitance between gate and substrate [13]. In contrast to the previous VMGFETs or suspended gate FETs, this paper introduces a simple

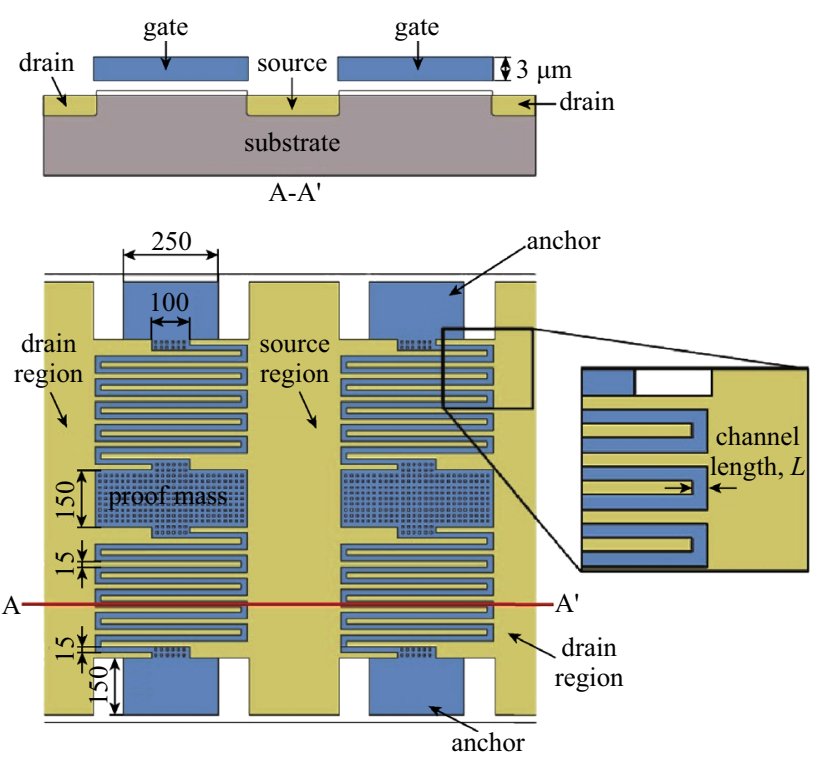

Fig. 1 Schematic of the vertically movable gate FET accelerometer (unit: $\mu \mathrm{m}$ ). Figure is not drawn to scale fabrication of VMGFET, which can be applied for lowfrequency vibration measurement. This paper describes the electrical properties of fabricated VMGFET and integration of VMGFET with electronics on printable circuit board (PCB), which can be transferred to the monolithic integration in the future.

\section{Fabrication}

The process details have been previously described [20]. The basic process to fabricate the VMGFETs consists of two UV lithography steps for silicon dry etching mask and an ion implantation mask shown schematically in Fig. 2. The VMGFETs are prepared using (100) oriented SOI wafers with a $3-\mu \mathrm{m}$-thick n-type device layer, a $1-\mu \mathrm{m}$-thick buried oxide (BOX) layer, and a 500- $\mu \mathrm{m}$-thick p-type handle layer. The p-type substrate is selected to form an n-channel MOSFET device. A 3- $\mu$ m-thick top device layer

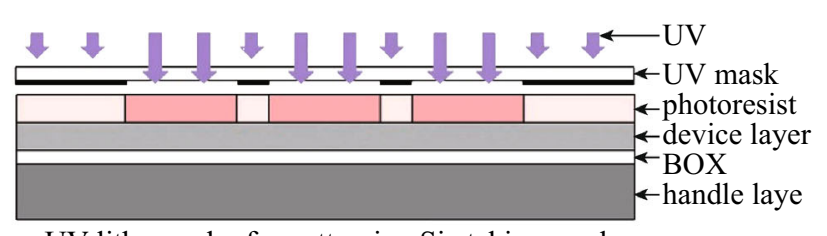

UV lithography for patterning Si etching mask

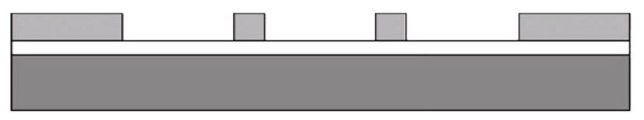

Si etching using ICP-RIE

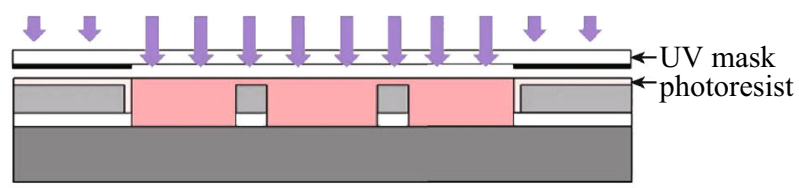

UV lithography for patterning ion implantation mask

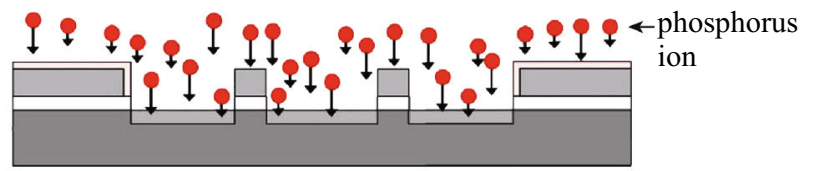

Ion implantation to form source and drain regions

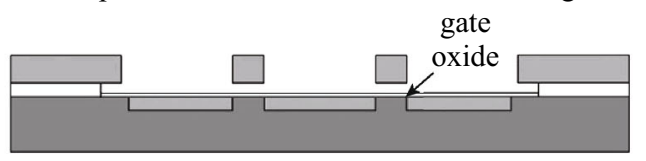

Releasing gate structures and gate oxide growth

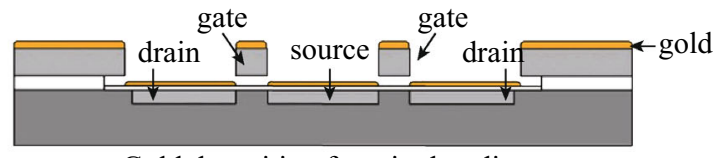

Gold deposition for wire bonding

Fig. 2 Schematic of fabrication process for the VMGFET accelerometer 
of SOI wafer is used to fabricate gate structures. The SOI device layer is patterned with the first UV mask using standard UV lithography. The wafer is etched through the device layer to the BOX using ICP-RIE process. A $1-\mu \mathrm{m}$ thick BOX layer is then opened and UV patterned using the second UV mask for ion implantation mask patterns. Then, phosphorus ions are implanted in the opened gate structures and the opened p-type substrate to form source and drain regions. Vapor HF etches the sacrificial layer underneath gate structures followed by thermal oxidation on the entire wafer to grow a 33-nm-thick gate oxide of the FET. A 200-nm-thick gold film is deposited on top of the wafer for wire bonding. Figure 3a shows an SEM image of fabricated dual VMGFETs with a common source region. The close-up view of suspended gate details is shown in Fig. 3b.
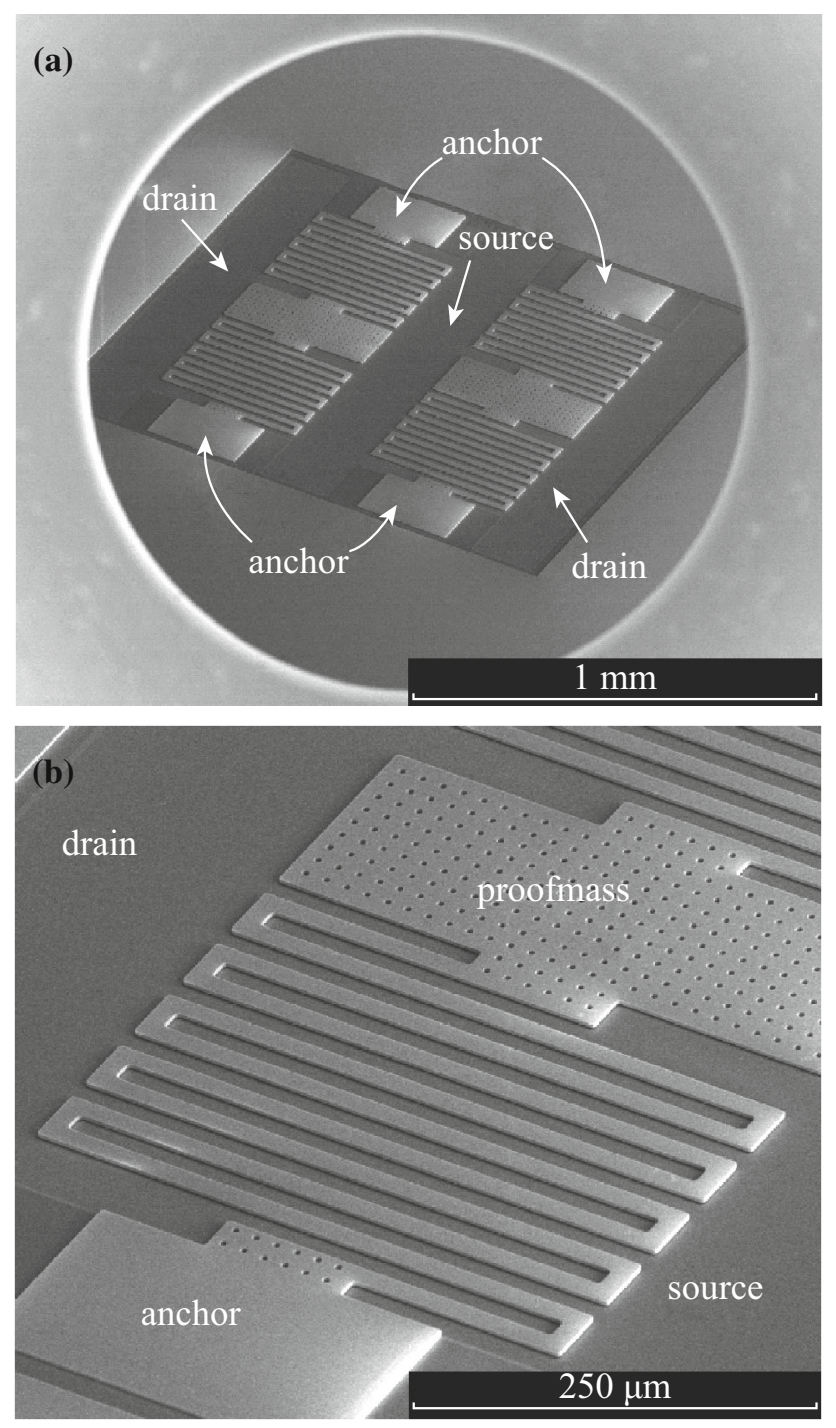

Fig. 3 a SEM image of fabricated dual VMGFETs. b The close-up view of suspended serpentine gate

\section{Mechanical Analysis}

A commercial finite element code, ABAQUS CAE 6.10-1, is used to build a finite element (FE) model and to investigate vibration behavior of the serpentine gate for design verification. Figure 4 shows the $\mathrm{FE}$ analysis results obtained assuming that the gate is a homogeneous medium made by silicon-gold composite and the anchors at the end of the gate are defined as fixed constraints. The gate structure is discretized into a finite number of elements in triangular and rectangular shape. Material properties of analysis model referred for the coated gold and silicon are summarized in Table 1. Coating layer of gold is modeled as uniformly distributed mass over the surface of the moving gate, ignoring additional stiffness from bonding interface between the two materials. The FE analysis performs eigenvalue extraction to calculate the natural frequencies and the corresponding mode shapes of the structure. As shown in Fig. 4, the fundamental mode shape of the gate structure is a typical bending mode of plates. The first natural frequency at the mode is obtained at $1799 \mathrm{~Hz}$. The second mode is a twist mode at $2059 \mathrm{~Hz}$, the third mode is the second bending at $4334 \mathrm{~Hz}$, and the fourth mode is the second twist at $5831 \mathrm{~Hz}$, and the fifth mode is the third bending mode at $6651 \mathrm{~Hz}$. The first natural frequency, therefore, is much higher than the applicable range of MEMS sensor of $500 \mathrm{~Hz}$, so that the natural frequencies of the structures of the sensor itself can hardly be affected by the measuring signal.

Twofold of gravity $(2 \mathrm{~g})$ is set as the target base acceleration for the developing sensor. The ground acceleration spectrum of the 1952 Kern County Earthquake, which is commonly used as a reference signal for nuclear power plant design, and is strongest since the 1906 San Francisco earthquake, is selected to investigate the structural integrity of the sensor in vibration [21]. As observed commonly in seismic accidents, most of the energy narrowly spread out within $50 \mathrm{~Hz}$.

Figure 5 shows the displacement response spectrum of the sensing element corresponding to the 1952 Kern Country earthquake spectrum. The first two peaks are the seismic source corresponding spectrum and the first bending mode at the first natural frequency at $10 \mathrm{~Hz}$ and $1799 \mathrm{kHz}$, respectively. The movements of sensing element from the neutral position corresponding to the two peaks in the response spectrum can reach to 0.1 and $1 \mu \mathrm{m}$. Figure 6a demonstrates the stress values under the 1952 Kern County Earthquake. Figure $6 \mathrm{~b}$ shows the stress level and stress field of the structure at $1799 \mathrm{~Hz}$ during vibration. The highest stress of $1.1 \mathrm{MPa}$, which is way below the material strength limit of sensing element, occurs at the first inner edge of the serpentine beam from both the anchors. 


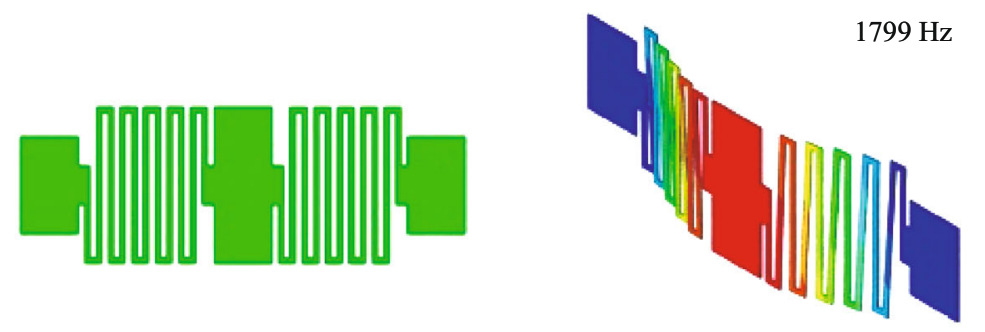

1 st mode

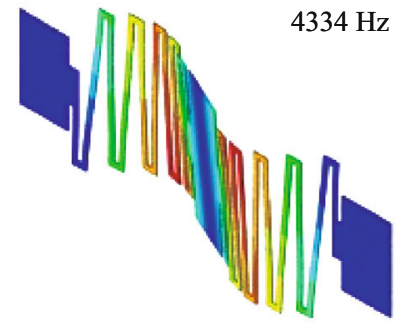

3rd mode

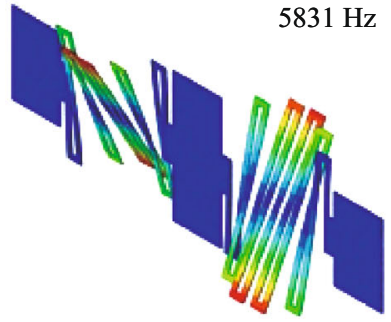

4th mode

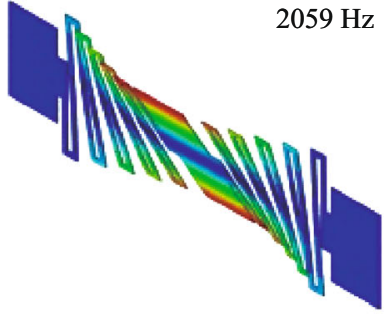

2nd mode

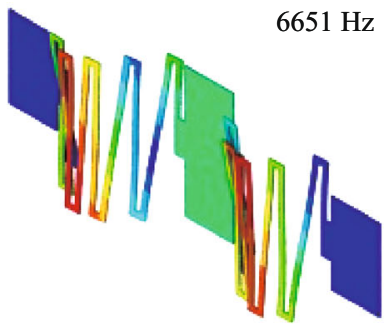

5th mode

Fig. 4 Finite element model and analysis results of the serpentine gate for the first five resonant frequency modes assuming a composite material as silicon-gold

Table 1 Material properties for gold and silicon for numerical model

\begin{tabular}{lll}
\hline Material & Property & \\
\hline Gold & Density & $19.2 \mathrm{~g} \mathrm{~cm}^{-3}$ \\
& Measured thickness & $0.292 \mu \mathrm{m}$ \\
Silicon & Density & $2.329 \mathrm{~g} \mathrm{~cm}^{-3}$ \\
& Elastic modulus & $169 \mathrm{GPa}$ \\
& Poison ratio & 0.048 \\
\hline
\end{tabular}

\section{Electrical Analysis}

The gate electrode of VMGFET is formed with a $0.1 \Omega \mathrm{cm}$ resistivity n-type device layer of SOI wafer. A (100) oriented boron-doped handle layer of SOI wafer exhibits p-type conductivity with 1-20 $\Omega \mathrm{cm}$ used for a substrate of FET embedding source and drain regions. A 1- $\mu \mathrm{m}$-thick air gap separates the gate and the substrate. The air gap allows the gate structure of VMGFET to move in vertical direction when the external forces such as acceleration or pressure are applied to the system. The gate motion shifts the current of the FET resulting from the change in air gap. Hence, VMGFET converts acceleration to electrical forms such as current or voltage without any additional circuitry.

For an n-channel VMGFET, a simplified model based on long-channel FET theory gives the drain current, $I_{\mathrm{DS}}$, in non-saturation region as

$I_{\mathrm{DS}}=\frac{\mu_{n} W}{L} C_{\mathrm{eff}}^{\prime}\left[\left(V_{\mathrm{GS}}-V_{\mathrm{Th}}\right) V_{\mathrm{DS}}-\frac{1}{2} V_{\mathrm{DS}}^{2}\right]$,

and in saturation region as

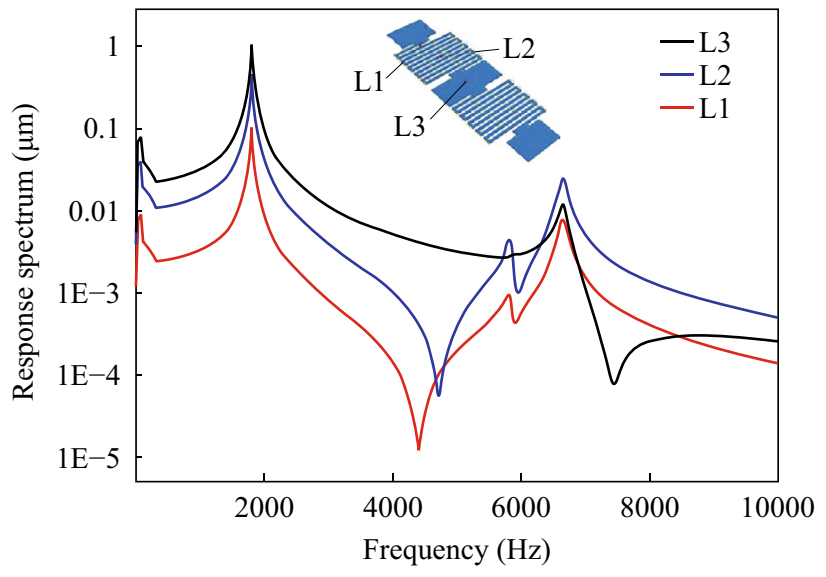

Fig. 5 Displacement spectrum corresponding to the ground acceleration spectrum at 1952 Kern County Earthquake in California USA

$I_{\mathrm{DS}}=\frac{\mu_{n} W}{2 L} C_{\mathrm{eff}}^{\prime}\left(V_{\mathrm{GS}}-V_{\mathrm{Th}}\right)^{2}$,

where $L$ is the channel length, $W$ is the gate width over the channel, and $\mu_{n}$ is channel carrier mobility. $V_{\mathrm{GS}}$ and $V_{\mathrm{DS}}$ are the potential differences of gate and drain to source, respectively. $V_{\mathrm{Th}}$ is the threshold voltage to form a channel between source and drain. $C_{\text {eff }}^{\prime}$ indicates an effective capacitance per unit area between gate electrode and silicon substrate consisting of two dielectrics: $C_{\mathrm{ox}}^{\prime}$ for gate oxide and $C_{\text {air }}^{\prime}$ for air. Hence, $C_{\text {eff }}^{\prime}$ is given by

$C_{\text {eff }}^{\prime}=\frac{C_{\mathrm{ox}}^{\prime} C_{\mathrm{air}}^{\prime}}{C_{\mathrm{ox}}^{\prime}+C_{\mathrm{air}}^{\prime}}=\frac{\varepsilon_{0} \varepsilon_{\mathrm{rox}}}{z_{\mathrm{gap}} \varepsilon_{\mathrm{rox}}+t_{\mathrm{ox}}}$, 


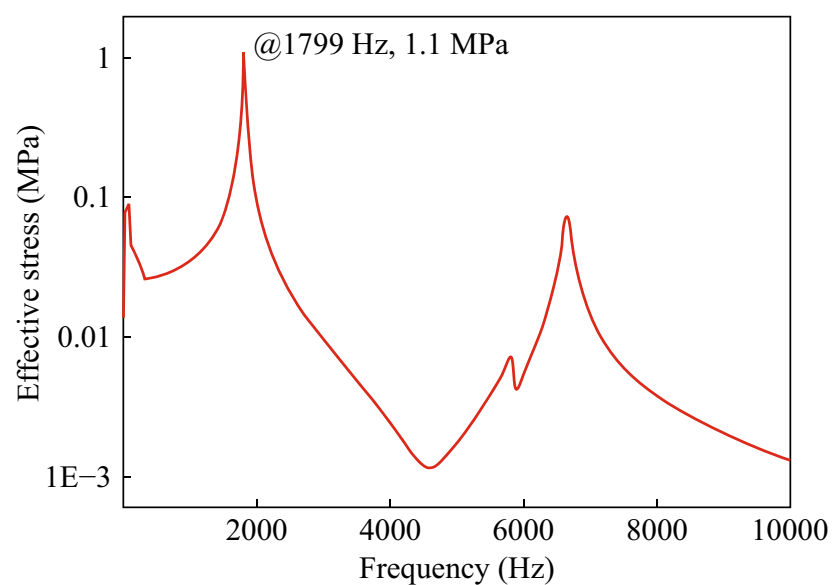

(a)

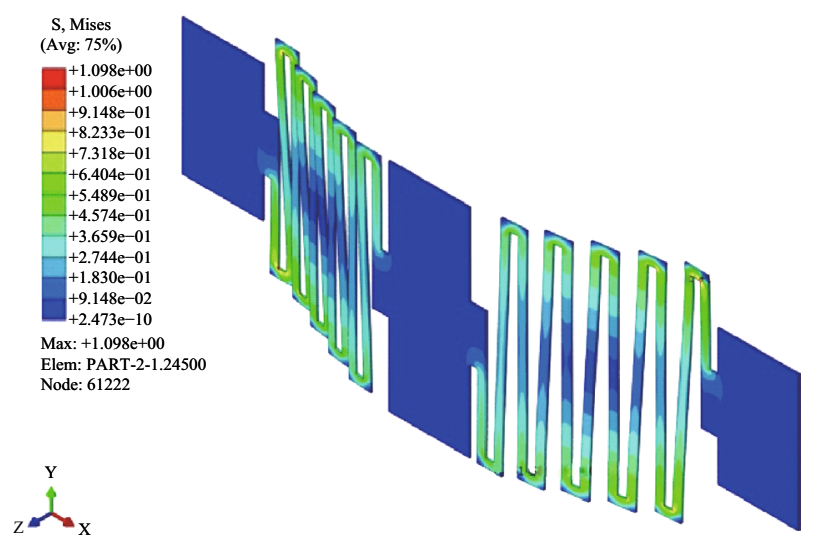

(b)

Fig. 6 a Stress spectrum corresponding to the displacement spectrum of the sensor. b Stress field on the serpentine gate calculated by FE analysis while the sensor suffers the 1952 Kern County Earthquake in California USA

where $\varepsilon_{\mathrm{rox}}=3.9$ is the relative dielectric permittivity of gate oxide, $\varepsilon_{0}=8.854 \times 10^{-14} \mathrm{~F} \mathrm{~cm}^{-1}$ is the permittivity of free space, $z_{\text {gap }}$ is the air gap between the bottom of the moving gate and top of the gate oxide layer over the channel, and $t_{\mathrm{ox}}$ is the thickness of the oxide. Since, as shown in Eq. (3), $C_{\text {eff }}^{\prime}$ is inversely proportional to the value of $z_{\text {gap }}$, the gate motion modulates the drain current in both non-saturation region and saturation region.Threshold voltage, $V_{\mathrm{Th}}$, of (1) and (2) is given by

$V_{\mathrm{Th}}=\phi_{\mathrm{MS}}+2 \phi_{\mathrm{F}}-\frac{\left(Q_{\mathrm{eff}}^{\prime}+Q_{\mathrm{d}}^{\prime}\right)}{C_{\mathrm{eff}}^{\prime}}$.

Here $\phi_{\mathrm{MS}}$ is the metal-semiconductor work function potential difference. The work functions in heavily doped n-type Si gate electrode and p-type Si substrate of $10 \Omega \mathrm{cm}$ resistivity are 4.58 and $5.03 \mathrm{eV}$, respectively [22]. Hence, $\phi_{\mathrm{MS}}$ of the fabricated VMGFET is $-0.45 \mathrm{~V}$. A negative value for $\phi_{\mathrm{MS}}$ means the gate of a MOS structure is positively charged and the silicon surface is negatively charged at equilibrium. For a p-type $\mathrm{Si}$ substrate, potential $\phi_{\mathrm{F}}$ is given by

$\phi_{\mathrm{F}}=\frac{k T}{q} \ln \frac{N_{\mathrm{A}}}{n_{i}}$,

where $q=1.6 \times 10^{-19} \mathrm{C}$ is magnitude of electron charge, $k=1.38 \times 10^{-23} \mathrm{~J} \mathrm{~K}^{-1}$ is the Boltzmann constant, $T$ is the temperature, and $n_{i}=1.5 \times 10^{10} \mathrm{~cm}^{-3}$ is the intrinsic doping concentration. The doping concentration, $N_{\mathrm{A}}$, of boron-doped p-type $\mathrm{Si}$ of $10 \Omega \mathrm{cm}$ resistivity is $1.5 \times 10^{15}$ $\mathrm{cm}^{-3}$, which determines $\phi_{\mathrm{F}}$ of $0.298 \mathrm{~V}$ using Eq. (5). The value for $\left(\phi_{\mathrm{MS}}+2 \phi_{\mathrm{F}}\right)$ term is $0.146 \mathrm{~V}$. $Q_{\mathrm{d}}^{\prime}$ is the depletion region charge per unit area in silicon substrate at onset of strong inversion given by $Q_{\mathrm{d}}^{\prime}=-\sqrt{4 \varepsilon_{s} q N_{\mathrm{A}} \phi_{\mathrm{F}}}$ for a p-type substrate. Here, $\varepsilon_{\mathrm{s}}=11.9$ is permittivity of silicon. $Q_{\mathrm{d}}^{\prime}$ for the given $N_{A}$ of $1.5 \times 10^{15} / \mathrm{cm}^{3}$ is $-1.736 \times 10^{-8} \mathrm{C} \mathrm{cm}^{-2}$. $Q_{\text {eff }}^{\prime}$ in Eq. (4) is an effective charge density in the gate oxide and at the oxide-silicon interface, which is extracted from experimental results in the following section.

\section{Experimental Results}

Figure 7 shows the electric measurement design of dual VMGFETs with a common source. The measured DC current-voltage $\left(I_{\mathrm{DS}}-V_{\mathrm{GS}}\right)$ characteristics of the prototype of VMGFET are shown in Fig. 8a for five different $V_{\mathrm{DS}}$ values. The threshold voltage of VMGFET extracted is $2.32 \mathrm{~V}$, which indicates that an enhancement-type n-channel device. $Q_{\text {eff }}^{\prime}$ of $1.545 \times 10^{-8} \mathrm{C} \mathrm{cm}^{-2}$ is extracted from measured $V_{\mathrm{Th}}(=2.32 \mathrm{~V})$ and $C_{\mathrm{eff}}^{\prime}\left(=8.780 \times 10^{-10}\right.$ $\mathrm{F} \mathrm{cm}^{-2}$ ) without gate motion under $1-\mu \mathrm{m}$ air gap using Eq. (4).

Figure $8 \mathrm{~b}$ shows the current-voltage $\left(I_{\mathrm{DS}}-V_{\mathrm{DS}}\right)$ characteristics of the fabricated n-channel VMGFET device for three different $V_{\mathrm{GS}}$ values. Using Eq. (2) in saturation region, the mutual transconductance, $g_{\mathrm{msat}}$, is given as

$$
\begin{aligned}
g_{\mathrm{msat}} & =\left.\left.\frac{\partial I_{\mathrm{DS}}}{\partial V_{\mathrm{GS}}}\right|_{V_{\mathrm{DS}} \geq V_{\mathrm{DSsat}}} \approx \frac{\Delta I_{\mathrm{DS}}}{\Delta V_{\mathrm{GS}}}\right|_{V_{\mathrm{DS}} \geq V_{\mathrm{DSsat}}} \\
& =\frac{\mu_{n} W}{L} C_{\mathrm{eff}}^{\prime}\left(V_{\mathrm{GS}}-V_{\mathrm{Th}}\right)=\frac{2 I_{\mathrm{DS}}}{V_{\mathrm{GS}}-V_{\mathrm{Th}}} .
\end{aligned}
$$

The measured $V_{\text {Th }}$ provides the value of $g_{\text {msat }}=6.59$ $\mathrm{mA} \mathrm{V}^{-1}$ from Fig. $8 \mathrm{~b}$ which shows $I_{\mathrm{DS}}=5.535 \mathrm{~mA}$ at $V_{\mathrm{DS}}=3 \mathrm{~V}$ and $V_{\mathrm{GS}}=4 \mathrm{~V}$.

The measured drain current in Fig. 8b is the result under gravity of the earth $(+1 \mathrm{~g})$. Then the sensor is inverted $180^{\circ}$ and experiences a negative gravity $(-1 \mathrm{~g})$. The measured $I_{\mathrm{DS}}$ under $-1 \mathrm{~g}$ is decreased to $5.487 \mathrm{~mA}$ at $V_{\mathrm{DS}}=3 \mathrm{~V}$ and $V_{\mathrm{GS}}=4 \mathrm{~V}$, which is $5.535 \mathrm{~mA}$ under $+1 \mathrm{~g}$. The test shows the current sensitivity of $24 \mu \mathrm{A} \mathrm{g}^{-1}$ at $V_{\mathrm{DS}}=3 \mathrm{~V}$ and $V_{\mathrm{GS}}=4 \mathrm{~V}$. 


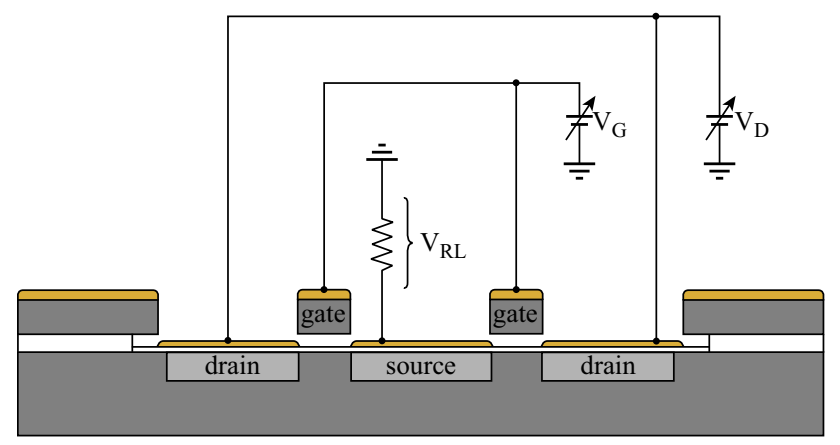

Fig. 7 Schematic diagram to measure the DC characteristics of fabricated VMGFET

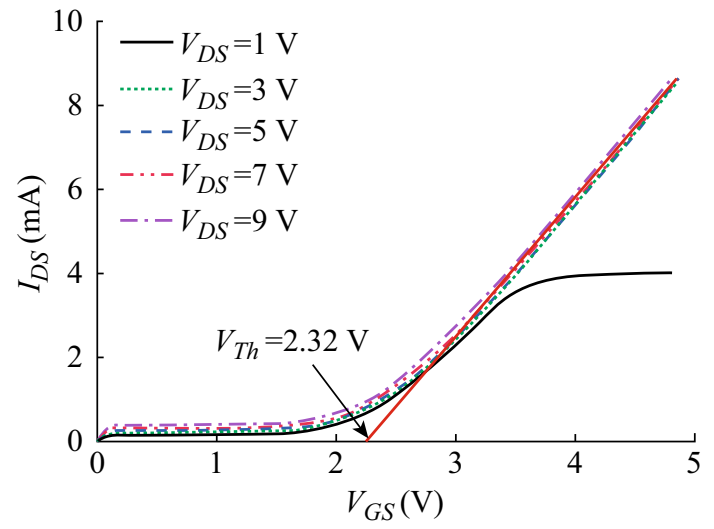

(a)

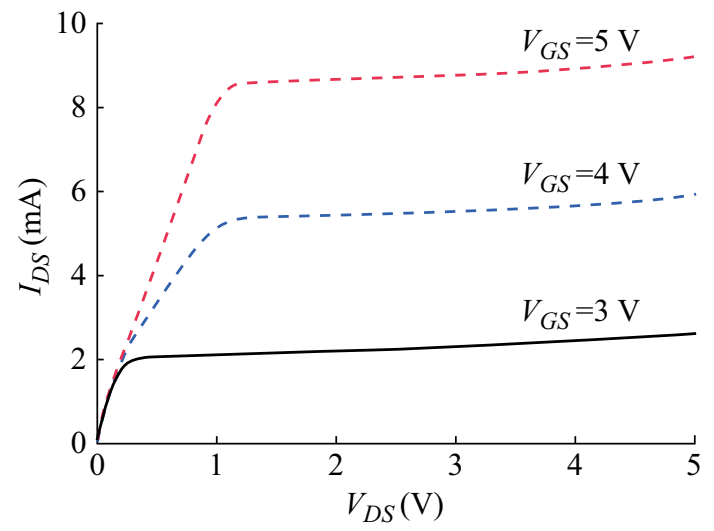

(b)

Fig. 8 a Measured DC $I_{\mathrm{DS}}-V_{\mathrm{GS}}$ characteristics with five different $V_{\mathrm{DS}}$. b Measured DC $I_{\mathrm{DS}}-V_{\mathrm{DS}}$ characteristics with three different $V_{\mathrm{GS}}$

Figure 9 is a simulation result based on (3) and (4) as a function of air gap distance, $z_{\mathrm{gap}}$. For this simulation, the following conditions are used: $N_{\mathrm{A}}=1 \times 10^{15} / \mathrm{cm}^{3}$, $t_{\mathrm{ox}}=33 \mathrm{~nm}, \quad Q_{\mathrm{d}}^{\prime}=-1.736 \times 10^{-8} \mathrm{C} \mathrm{cm}^{-2}$, and $Q_{\mathrm{eff}}^{\prime}=$ $1.545 \times 10^{-8} \mathrm{C} \mathrm{cm}^{-2}$. As shown in Fig. $9, V_{\mathrm{Th}}$ is not constant and linearly decreased as the gate is closed to the substrate. With the decreasing air gap, the change in $V_{\mathrm{Th}}$ of the fabricated VMGFET modulates the drain current in both non-saturation and saturation regions. The value of $\left(\mu_{n} W(2 L)^{-1}\right)$ in Eq. (2) can be extracted from Fig. 8 b for the evaluation of device motion sensitivity. The drain current is $5.535 \mathrm{~mA}$ at $V_{\mathrm{DS}}=3 \mathrm{~V}$ and $V_{\mathrm{GS}}=4 \mathrm{~V}$ for $1-\mu \mathrm{m}$ air gap. The threshold voltage and the effective capacitance are $2.32 \mathrm{~V}$ and $8.854 \times 10^{-10} \mathrm{~F} / \mathrm{cm}^{2}$, respectively. Hence, the value of $\left(\mu_{n} W / 2 L\right)$ is $2.2375 \times 10^{6} \mathrm{~cm}^{2}$ $(\mathrm{V} \mathrm{s})^{-1}$ from Eq. (2). Figure 9 also shows the simulation of drain current as a function of air gap thickness at $V_{\mathrm{DS}}=3 \mathrm{~V}$ and $V_{\mathrm{GS}}=4 \mathrm{~V}$. The simulation is based on (2). For example, the $50 \mathrm{~nm}$ gate displacement down from the original position increases the drain current to $5.634 \mathrm{~mA}$.

The output voltage of VMGFET is measured using readout circuit integrated on a printed circuit board (PCB) to detect voltage changes. Figure 10 illustrates the block diagram of readout circuit and displacement detection circuit with filter and amplifier. The gates move toward or backward the substrate when the acceleration is applied to the common source VMGFETs. The voltage across the load resistor is filtered and amplified. The readout circuit outputs a voltage proportional to the displacement of gate from steady state position. The experiments with external acceleration are performed on a setup comprising a platform with a fixed stage on a vibration exciter as shown in Fig. 11. The shaker actuates the suspended gate vertically since the gate motion is out of plane and the sensitivity axis is aligned with the platform's vertical direction.

Figure 12 shows the relation of input acceleration to output voltage for different actuation frequencies. According to the results, the device shows the linear feedback voltage sensitivity of $9.36-9.42 \mathrm{mV} \mathrm{g}^{-1}$ in the measurement range of $0.5-1.2 \mathrm{~g}$ for the actuated frequencies. The frequency response of vibration measurement provides the flatness of measuring signal along the operating frequency range and the location of the first natural frequency, which determines

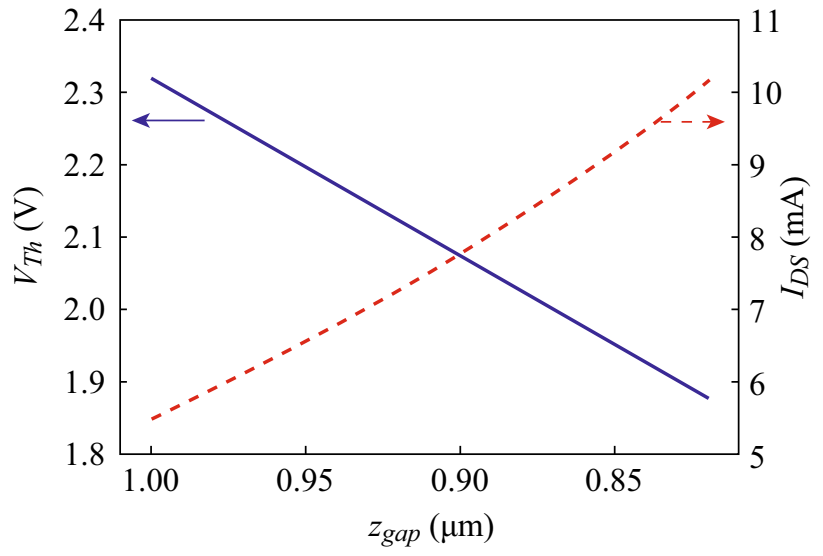

Fig. 9 Simulated threshold voltage, $V_{\mathrm{Th}}$, of the prototype VMGFET based on (3) and (4) and simulated drain current, $I_{\mathrm{DS}}$, at $V_{D S}=3 \mathrm{~V}$ and $V_{\mathrm{GS}}=4 \mathrm{~V}$ as a function of air gap thickness, $z_{\mathrm{gap}}$ 
the operating frequency range of the fabricated VMGFET. The frequency response of the fabricated device and the flatness of the signal are measured using SC-1000 Sine vibration controller (IMV Corp., Japan) as shown in Fig. 13. It shows the first natural frequency of the tested device at $1230 \mathrm{~Hz}$ which is a lower resonant frequency than that of simulation. As shown in Fig. 13, the profile smoothness of the sensed signal is in $3 \mathrm{~dB}$ range up to $1 \mathrm{kHz}$. Under a $1 \mathrm{~g}$ $\left(=9.8 \mathrm{~m} \mathrm{~s}^{-2}\right)$ acceleration, the sensed accelerations are 9.78 and $9.15 \mathrm{~m} \mathrm{~s}^{-2}$ at 10 and $500 \mathrm{~Hz}$, respectively.

\section{Conclusion}

A VMGFET is designed and fabricated for a micro-accelerometer, which converts the acceleration to electrical signals. In order to form VMGFET, SOI wafer is used to simplify the fabrication processes. Prior to the fabrication and electrical measurement, a finite element analysis is carried out to evaluate mechanical feasibility and structural integrity as a sensing element. Free vibration analysis shows that the fundamental natural frequency of the gate structure is $1799 \mathrm{~Hz}$ whose corresponding mode shape is a typical bending mode of a plate. In this paper, the maximum base excitation for seismic response spectrum analysis is twofold of gravity ( $2 \mathrm{~g}$ ). The ground acceleration spectrum of the 1952 Kern County Earthquake, commonly

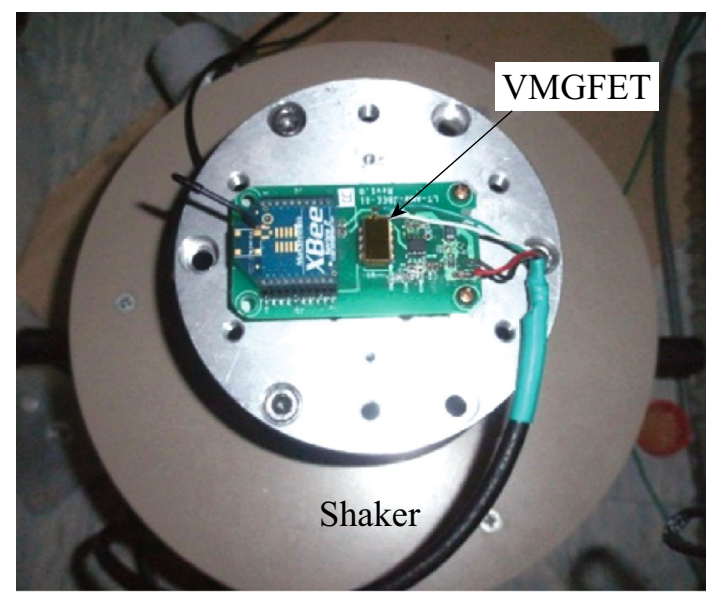

Fig. 11 Experimental setup mounted on shaker to apply vibrations to the prototype VMGFET integrated on PCB

used as the reference excitation signal for nuclear power plant design, is selected to investigate the structural integrity of the sensor in vibration. Highest stress, at the first inner edge of the serpentine beam from both the anchors, from seismic excitation is way below the material strength limit.

By DC electrical measurement, the threshold voltage and the effective charge density in the gate oxide and at the interface between silicon and gate oxide are determined of $2.32 \mathrm{~V}$ and $1.545 \times 10^{-8} \mathrm{C} \mathrm{cm}^{-2}$, respectively. A readout
Fig. 10 Schematic circuit diagram to measure frequency analysis and device sensitivity

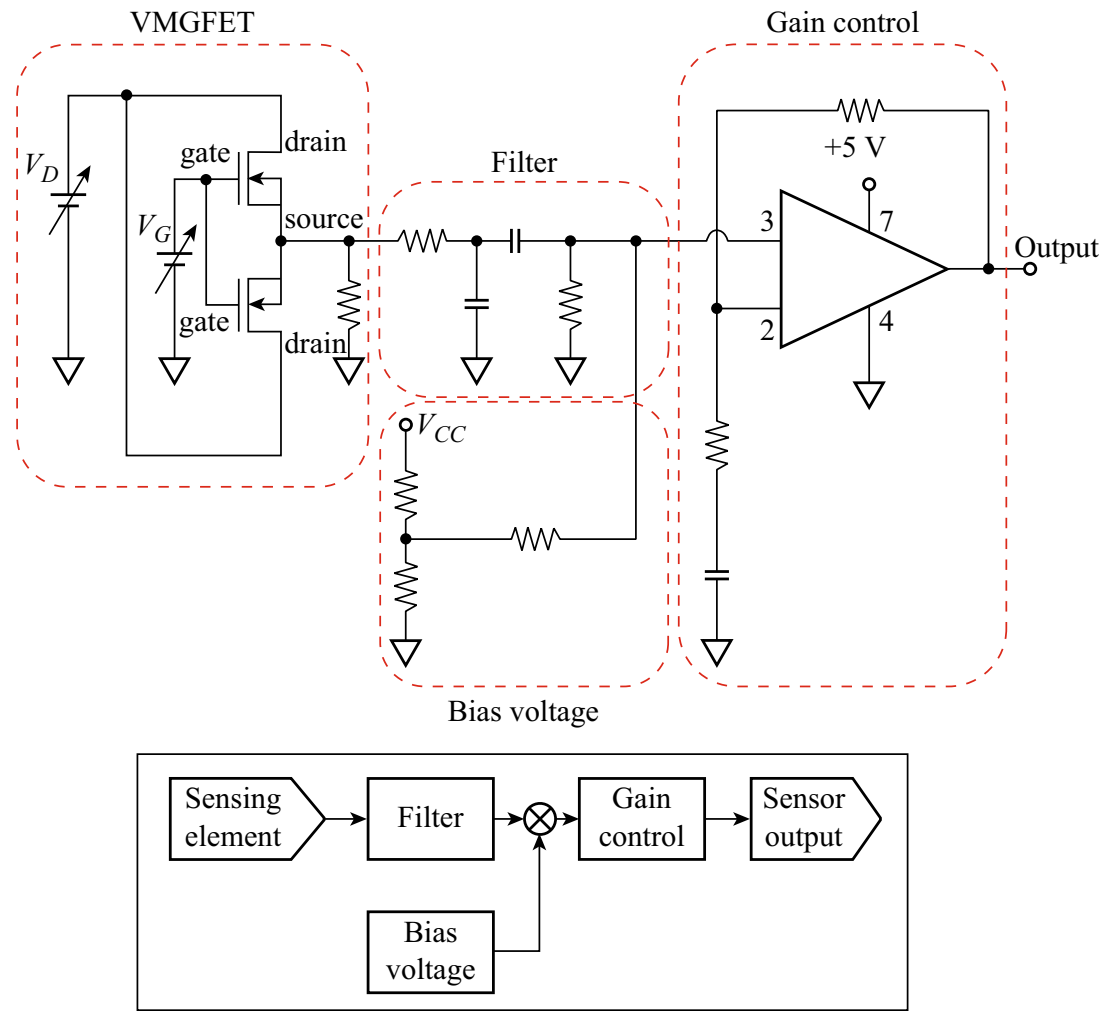




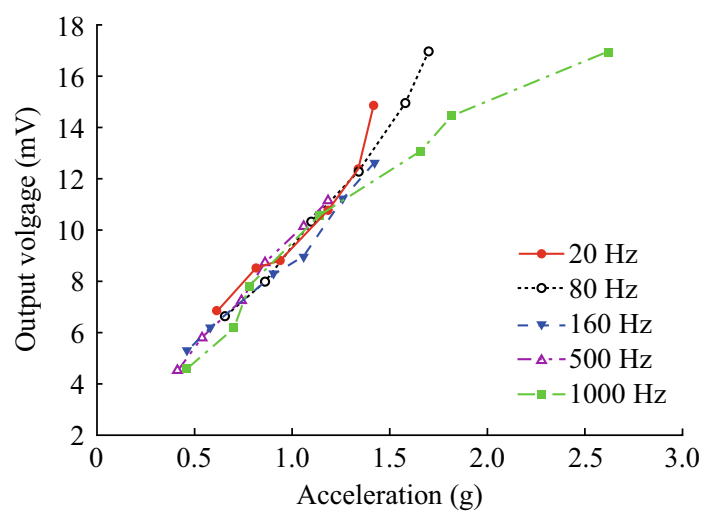

Fig. 12 Output response to applied accelerations for different frequencies

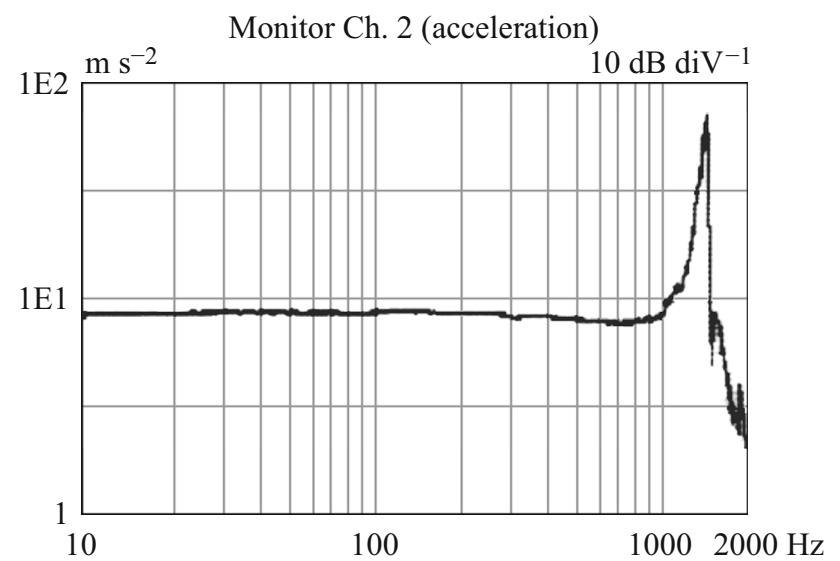

Fig. 13 Frequency response of the prototype VMGFET. The resonant frequency is at $1.23 \mathrm{kHz}$

circuit is designed and integrated on PCB with VMGFET. The voltage sensitivity of the device is $9.36-9.42 \mathrm{mV} \mathrm{g}^{-1}$ up to $1000 \mathrm{~Hz}$. According to the frequency spectrum, the first natural frequency of the tested device is located at $1230 \mathrm{~Hz}$, which is lower than that of the simulation result. The difference between the simulation and experiment can be due to the actual thickness of the device layer being thinner than that used for the simulation and the deposited gold film thickness being thicker, which does not provide accurate parameter information for the simulation. The prototype VMGFET shows that the smoothness of the sensed signal profile is in $3 \mathrm{~dB}$ range up to $1 \mathrm{kHz}$. Under a $1 \mathrm{~g}\left(=9.8 \mathrm{~m} \mathrm{~s}^{-2}\right)$ acceleration, the sensed accelerations are 9.78 and $9.15 \mathrm{~m} \mathrm{~s}^{-2}$ at 10 and $500 \mathrm{~Hz}$, respectively. Seismic load is narrowly distributed in the frequency range of 0 to $40 \mathrm{~Hz}$. For the application of seismic monitoring in nuclear power plants, the accelerometer would be sensitive and reliable in the low frequency range. Hence, the prototype VMGFET is suitable for the acceleration measurement of the low-frequency seismic vibrations.
Acknowledgments This work was supported by the National Research Foundation of Korea (NRF) funded by the Korea government (MSIP) (No. 2012M2A8A5025825). Authors would like to acknowledge and thank Daedeok INNOPOLIS Foundation.

Open Access This article is distributed under the terms of the Creative Commons Attribution 4.0 International License (http:// creativecommons.org/licenses/by/4.0/), which permits unrestricted use, distribution, and reproduction in any medium, provided you give appropriate credit to the original author(s) and the source, provide a link to the Creative Commons license, and indicate if changes were made.

\section{References}

1. M.G. Bardon, H.P. Neves, R. Puers, C.V. Hoof, Scaling the suspended-gate FET: impact of dielectric charging and roughness. IEEE Trans. Electron Devices 57, 804-813 (2010). doi:10. 1109/TED.2009.2039963

2. K. Scharnagl, M. Bogner, A. Fuchs, R. Winter, T. Doll, I. Eisele, Enhanced room temperature gas sensing with metal oxides by means of the electroadsorptive effect in hybrid suspended gate FET. Sens. Actuators, B 57, 35-38 (1999). doi:10.1016/S09254005(99)00059-3

3. A.-C. Salaun, F. Le Bihan, T. Mohammed-Brahim, Modeling the high $\mathrm{pH}$ sensitivity of suspended gate field effect transistor (SGFET). Sens. Actuators, B 158, 138-143 (2011). doi:10.1016/j. snb.2011.05.056

4. A. Persano, A. Cola, G. De Angelis, A. Taurino, P. Siciliano, F. Quaranta, Capacitive RF MEMS switches with tantalum-based materials. J. Microelectromech. Syst. 20, 365-370 (2011). doi:10. 1109/JMEMS.2011.2107884

5. J.-I. Lee, X. Huang, P.B. Chu, Nanoprecision MEMS capacitive sensor for linear and rotational positioning. J. Microelectromech. Syst. 18, 660-670 (2009). doi:10.1109/JMEMS.2009.2016275

6. X. Sun, X. Peng, Y. Zheng, X. Li, H. Zhang, A 3-D Stacked highQ PI-based MEMS inductor for wireless power transmission system in bio-implanted applications. J. Microelectromech. Syst. 23, 888-898 (2014). doi:10.1109/JMEMS.2013.2297627

7. V. Lemarquand, G. Lemarquand, E. Lefeuvre, I. Shahosseini, R. Ravaud, J. Moulin, M. Woytasik, E. Martinsic, G. Pillonnet, Electrodynamic MEMS: application to mobile phone loudspeakers. IEEE Trans. Magn. 48, 3684-3687 (2009). doi:10.1109/ TMAG.2012.2203798

8. F. Caimmi, S. Mariani, M. De Fazio, P. Bendiscioli, Investigation of the effectiveness and robustness of an mems-based structural health monitoring system for composite laminates. IEEE Sens. J. 14, 2208-2215 (2014). doi:10.1109/JSEN.2014.2315831

9. Y. Yee, J. Bu, K. Chun, J.-W. Lee, An integrated digital silicon micro-accelerometer with MOSFET-type sensing elements. J. Micromech. Microeng. 10, 350-358 (2000). doi:10.1088/0960$1317 / 10 / 3 / 308$

10. R.S. Jachowicz, Z.M. Azgin, FET pressure sensor and iterative method for modelling of the device. Sens. Actuators, A 97-98, 369-378 (2002). doi:10.1016/S0924-4247(01)00856-1

11. C.-L. Dai, P.-H. Kao, Y.-W. Tai, C.-C. Wu, Micro FET pressure sensor manufactured using CMOS-MEMS technique. Microelectron. J. 39, 744-749 (2008). doi:10.1016/j.mejo.2007.12.015

12. I.-H. Song, P.K. Ajmera, A laterally movable gate field effect transistor. J. Microelectromech. Syst. 18, 208-216 (2009). doi:10. 1109/JMEMS.2008.2008623

13. J.M. Williams, B.S. Cole, B.H. You, H.S. Kang, I.-H. Song, Electrical modelling and design insight of a vertically movable gate field effect transistor for physical sensor applications. IET 
Micro Nano Lett. 7, 1117-1120 (2012). doi:10.1049/mnl.2012. 0667

14. G. Langfelder, T. Frizzi, A. Longoni, A. Tocchio, D. Manelli, E. Lasalandra, Readout of MEMS capacitive sensors beyond the condition of pull-in instability. Sens. Actuators, A 167, 374-384 (2011). doi:10.1016/j.sna.2011.02.003

15. A.L. Roy, H. Sarkar, A. Dutta, T.K. Bhattacharyya, A high precision SOI MEMS-CMOS $\pm 4 \mathrm{~g}$ piezoresistive accelerometer. Sens. Actuators, A 210, 77-85 (2014). doi:10.1016/j.sna.2014.01. 036

16. B.V. Amini, F. Ayazi, Micro-gravity capacitive silicon-on-insulator accelerometers. J. Micromech. Microeng. 15, 2113-2120 (2005). doi:10.1088/0960-1317/15/11/017

17. S. Aoyagi, M. Suzuki, J. Kogure, T. Kong, R. Taguchi, T. Takahashi, S. Yokoyama, H. Tokunaga, Accelerometer using MOSFET with movable gate electrode: Electroplating thick nickel proof mass on flexible parylene beam for enhancing sensitivity, in Technical Digest of the 16th International Conference on Solid-State Sensors, Actuators and Microsystems (Transducers 2011), Beijing (Jun 5-9, 2011), 2030-2033 (2011). doi:10.1109/ TRANSDUCERS.2011.5969210
18. K. Zandi, J.A. Belanger, Y.-A. Peter, Design and demonstration of an in-plane silicon-on-insulator optical MEMS fabry-perotbased accelerometer integrated with channel waveguides. J. Microelectromech. Syst. 21, 1464-1470 (2012). doi:10.1109/ JMEMS.2012.2211577

19. S.E. Alper, Y. Temiz, T. Akin, A compact angular rate sensor system using a fully decoupled silicon-on-glass MEMS gyroscope. J. Microelectromech. Syst. 17, 1418-1429 (2008). doi:10. 1109/JMEMS.2008.2007274

20. I.-H. Song, B. You, H. Kang, K.-H. Lee, A vertically movable gate field effect transistor (VMGFET) on a silicon-on-insulator (SOI) wafer and method of forming a VMGFET. PCT/US2013/ 064239 (Oct 4, 2013)

21. K. Hutton, Earthquake monitoring in Southern California for seventy-seven years (1932-2008). Bull. Seismol. Soc. Am. 100, 423-446 (2010)

22. A. Novikov, Experimental measurement of work function in doped silicon surfaces. Solid-State Electron. 54, 8-13 (2010). doi:10.1016/j.sse.2009.09.005 